\title{
DVCS measurements with nuclear targets at HERMES
}

\author{
M. Hoek \\ University of Giessen \\ on behalf of the \\ HERMES collaboration \\ Lake Louise Winter Institute 2006

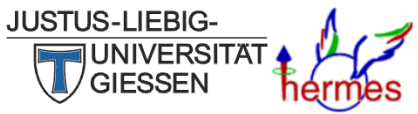




\section{Generalised Parton Distributions}

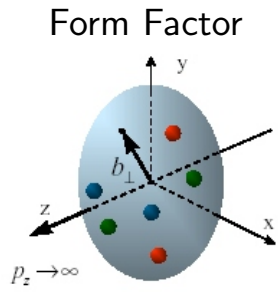

Structure Function
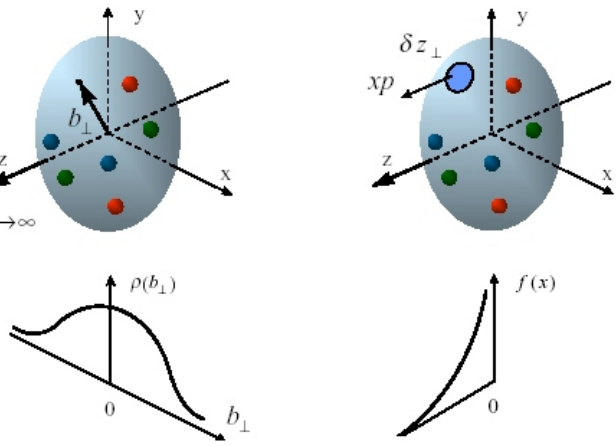
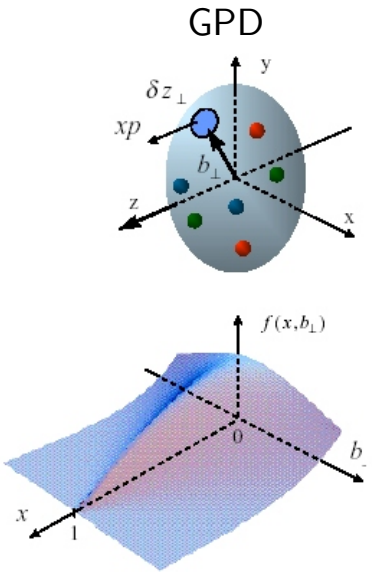


\section{Generalised Parton Distributions}

- Accessible in exclusive reactions

$\rightarrow$ Simplest process : $\gamma^{*} p \rightarrow p^{\prime} \gamma$

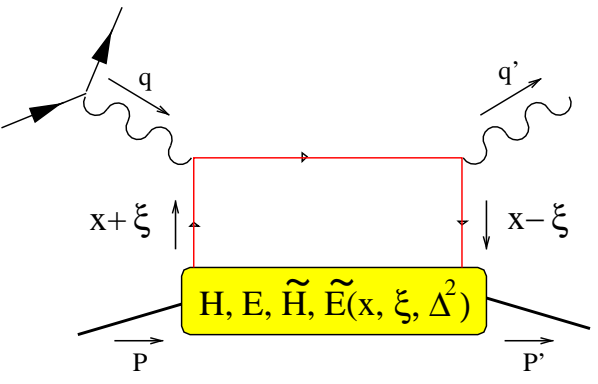

- Constrained by FF \& Structure Function

- Depend on three variables

- $\mathrm{x}, \xi \rightarrow$ light-cone momentum fractions

- $\xi \rightarrow \frac{x_{B}}{2-x_{B}}$ in Bjorken limit

- $\Delta^{2}=\left(p_{\gamma}^{*}-p_{\gamma}\right)^{2}=-t$

DVCS on proton target

- Nuclear spin determines number of GPDs
- $\mathrm{I}=0 \rightarrow 1 \mathrm{GPD}$
- $\mathrm{I}=1 / 2 \rightarrow 4$ GPDs
- $\mathrm{I}=1 \rightarrow 9$ GPDs 


\section{Deeply Virtual Compton Scattering}

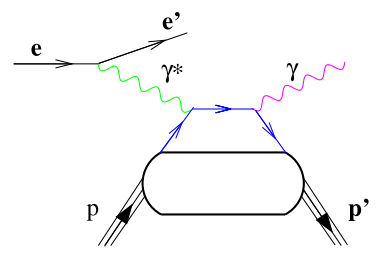

DVCS

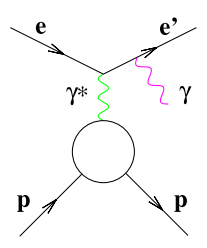

Bethe-Heitler
- DVCS-BH interference

- BH dominates at HERMES kinematics

- Decompose $\mathcal{I}$ in sum of Fourier harmonics

(Belitsky et al. hep-ph/0112108)

- Beam Charge Asymmetry $\rightarrow \cos \phi$ behaviour

- Beam Spin Asymmetry

$\rightarrow \sin \phi$ behaviour $\rightarrow$ beam polarisation $\mathrm{P}_{\text {I }}$

Photon production $d \sigma \propto \underbrace{\left|\tau_{B H}\right|^{2}}_{Q E D}+\left|\tau_{D V C S}\right|^{2}+\overbrace{\tau_{D V C S} \tau_{B H}^{*}+\tau_{D V C S}^{*} \tau_{B H}}^{\mathcal{I}}$ 


\section{Deeply Virtual Compton Scattering}

- DVCS-BH interference

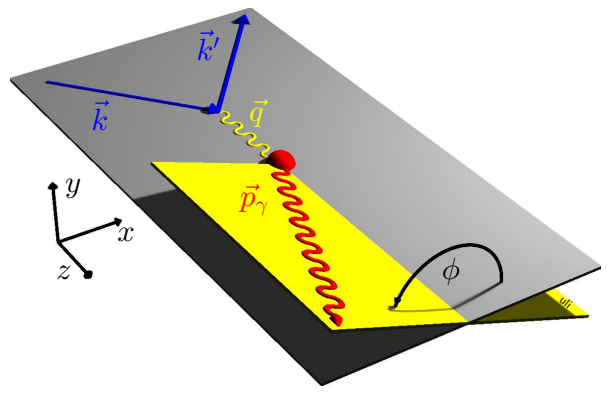

- BH dominates at HERMES kinematics

- Decompose $\mathcal{I}$ in sum of Fourier harmonics

(Belitsky et al. hep-ph/0112108)

- Beam Charge Asymmetry $\rightarrow \cos \phi$ behaviour

- Beam Spin Asymmetry $\rightarrow \sin \phi$ behaviour $\rightarrow$ beam polarisation $P_{\text {I }}$

$$
\mathcal{I}= \pm \frac{4 \sqrt{2} m e^{6}}{t Q x_{B}} \frac{1}{\sqrt{1-x_{B}}} \cdot \cos \phi \frac{1}{\epsilon(\epsilon-1)} \Re M^{1,1}-P_{/} \sin \phi \sqrt{\frac{1+\epsilon}{\epsilon}} \Im M^{1,1}
$$




\section{The HERA Acclerator at DESY}

$\mathrm{e}^{ \pm}$beam: $27.6 \mathrm{GeV} / \mathrm{c}$

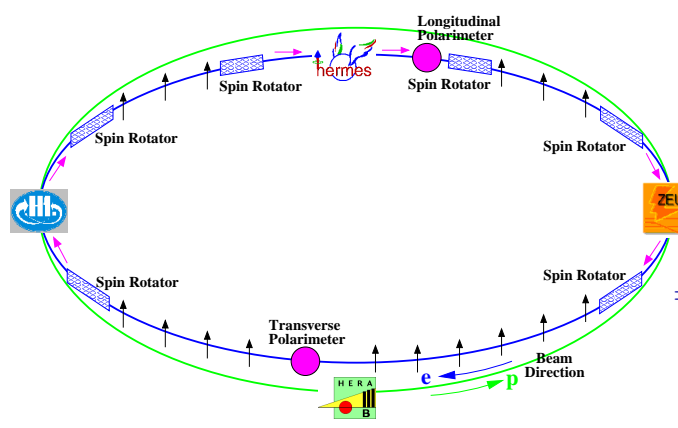

proton beam: $920 \mathrm{GeV} / \mathrm{c}$
- Collider experiments: ZEUS \& H1

- Fixed target experiments: HERMES \& HERA B

- Synchrotron Radiation

$\Rightarrow$ Transverse Polarisation (Sokolov-Ternov-Effect)

- Polarisation up to $60 \%$

- Spin rotators

$\Rightarrow$ Longitudinal Polarisation 


\section{The HERMES Spectrometer}

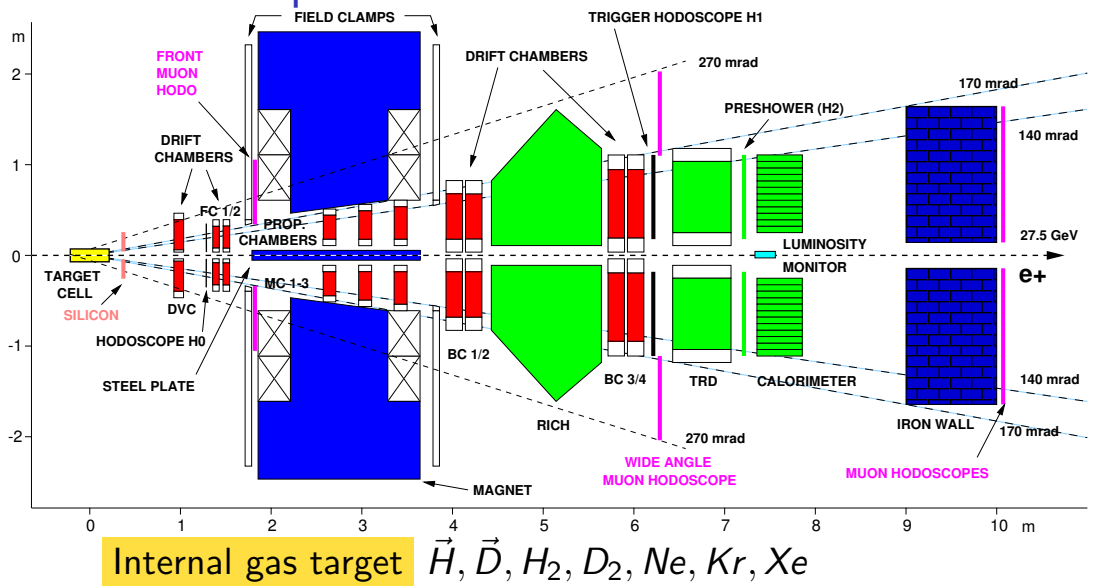

Tracking $\frac{\Delta P}{P}<1.6 \%$

Particle Identification $\epsilon_{e^{ \pm}} \geq 97 \%$

M. Hoek

DVCS measurements with nuclear targets at HERMES 


\section{Exclusive Event Selection}
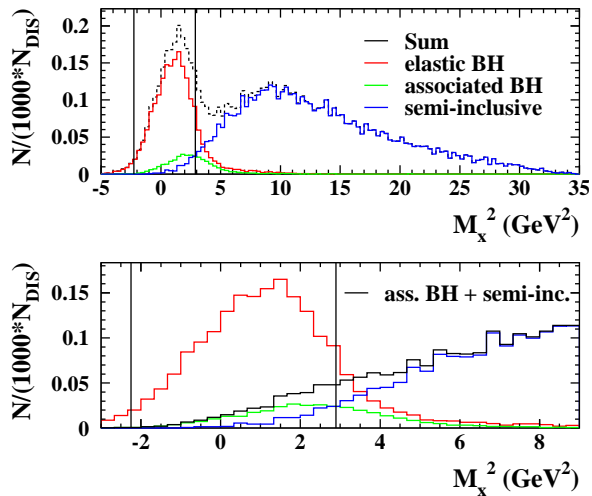

$$
M_{x}^{2}=m_{p}^{2}+2 m_{p}\left(\nu-E_{\gamma}\right)+t
$$

- Events with 1 DIS-Electron or - positron and one trackless

\section{cluster}

- Kinematic requirements

$$
\begin{aligned}
& \rightarrow \mathrm{Q}^{2}>1 \mathrm{GeV}^{2} \\
& \rightarrow \mathrm{W}^{2}>4 \mathrm{GeV}^{2}
\end{aligned}
$$

- Exclusivity via missing mass

$$
-1.5<\mathrm{M}_{X}<1.7 \mathrm{GeV}
$$

- Estimate background contribution from MC

- Associated BH/DVCS

$$
\left(e p \rightarrow e^{\prime} \Delta^{+} \gamma\right)
$$

- Semi-Inclusive

$$
\left(e p \rightarrow e^{\prime} \pi^{\circ} X\right)
$$

- Overall background contribution $\approx 15 \%$ 


\section{Beam Charge Asymmetry on Deuterium}
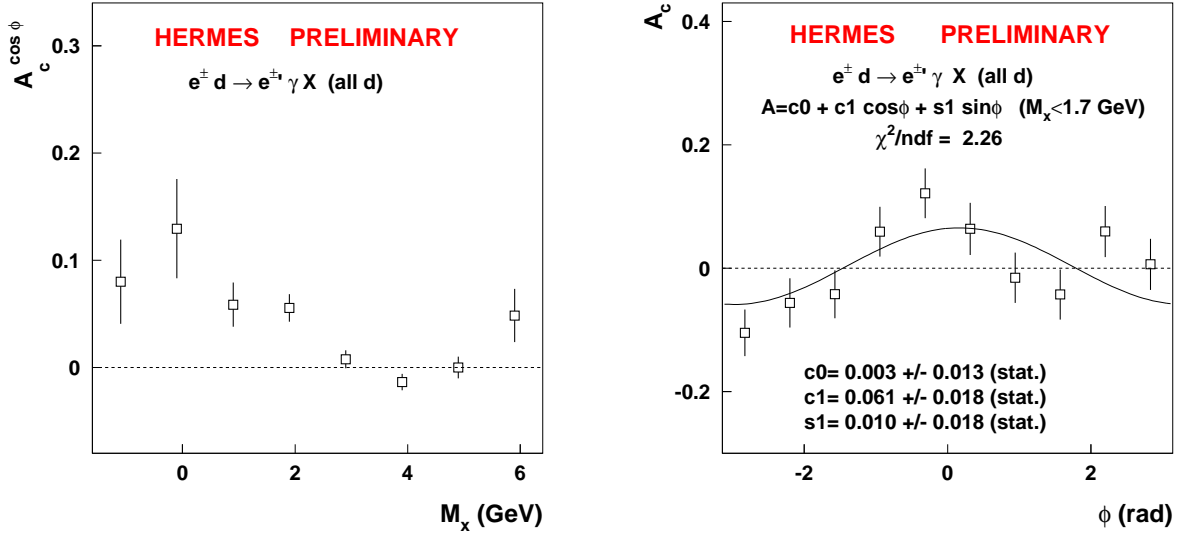

$A_{C}(\phi)=\frac{N^{+}(\phi)-N^{-}(\phi)}{N^{+}(\phi)+N^{-}(\phi)}$

- Expected $\cos (\phi)$ dependence

- $\cos (\phi)$-Moments vanishing at higher missing mass 


\section{Beam Spin Asymmetry on Nuclear Targets}

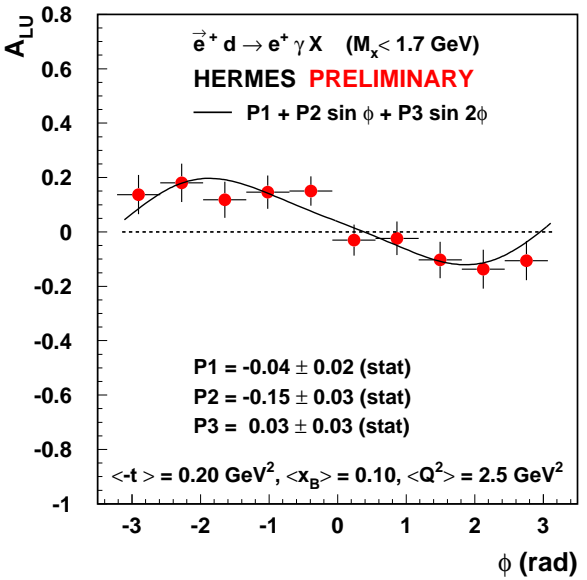

$A_{L U}(\phi)=\frac{1}{\left\langle\left|P_{B}\right|\right\rangle} \frac{\vec{N}(\phi)-\overleftarrow{N}(\phi)}{\vec{N}(\phi)+\overleftarrow{N}(\phi)}$
- Expected $\sin (\phi)$ dependence

- $\sin (\phi)$-Moments vanishing at higher missing mass

- Integrating over $t$

- Deuterium

- Spin 1

- Neon

- Spin 0

- Krypton

- Spin $0(9 / 2)$

$\rightarrow$ Admixture from other isotopes 


\section{Beam Spin Asymmetry on Nuclear Targets}

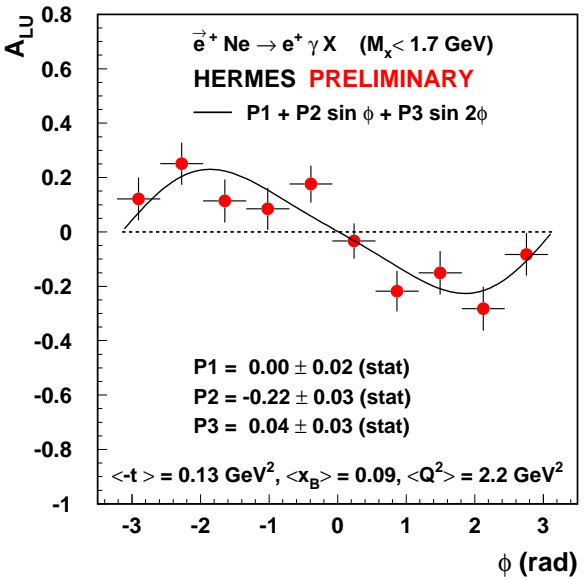

$A_{L U}(\phi)=\frac{1}{\left\langle\left|P_{B}\right|\right\rangle} \frac{\vec{N}(\phi)-\overleftarrow{N}(\phi)}{\vec{N}(\phi)+\overleftarrow{N}(\phi)}$
- Expected $\sin (\phi)$ dependence

- $\sin (\phi)$-Moments vanishing at higher missing mass

- Integrating over $t$

- Deuterium

- Spin 1

- Neon

- Spin 0

- Krypton

- Spin $0(9 / 2)$

$\rightarrow$ Admixture from other isotopes 


\section{Beam Spin Asymmetry on Nuclear Targets}

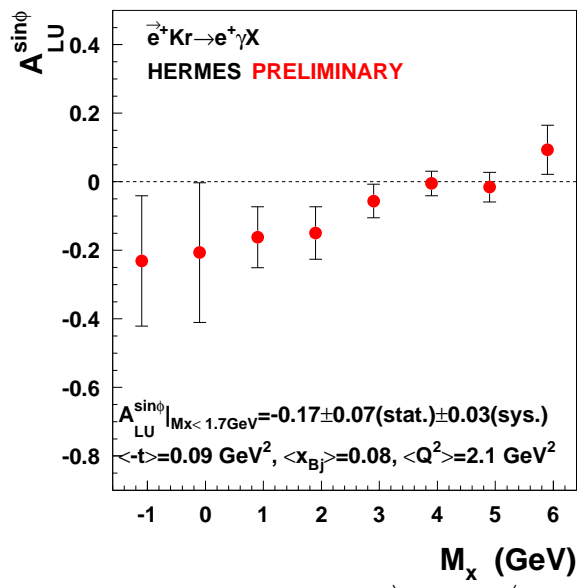

$A_{L U}(\phi)=\frac{1}{\left\langle\left|P_{B}\right|\right\rangle} \frac{\vec{N}(\phi)-\overleftarrow{N}(\phi)}{\vec{N}(\phi)+\overleftarrow{N}(\phi)}$
- Expected $\sin (\phi)$ dependence

- $\sin (\phi)$-Moments vanishing at higher missing mass

- Integrating over $\mathrm{t}$

- Deuterium

- Spin 1

- Neon

- Spin 0

- Krypton

- Spin $0(9 / 2)$

$\rightarrow$ Admixture from other isotopes 


\section{Predictions for Heavy Nuclei at HERMES}

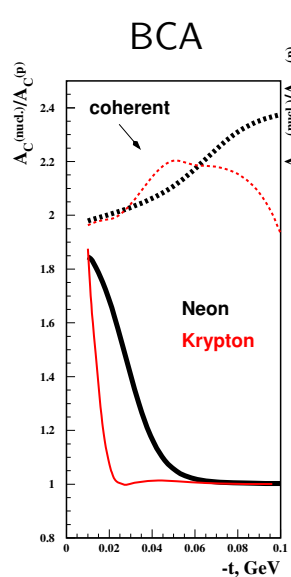

(Strikman hep-ph/0301216)
- Current results (-t $\left.<0.7 \mathrm{GeV}^{2}\right)$

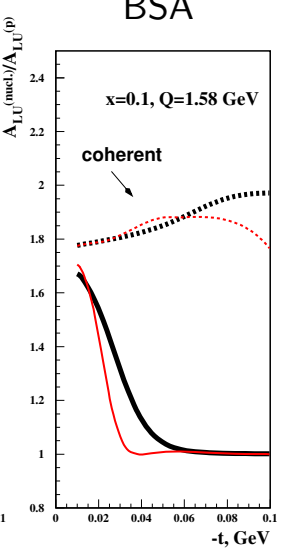

$\Rightarrow$ Need MC to estimate contamination 


\section{Conclusions}

- GPDs are a novel tool to describe the nucleon structure

$\rightarrow$ Access GPDs via exclusive processes (DVCS)

- Apply GPD formalism to nuclei

- 3D distributions of quarks and gluons inside nucleus

- Access to forces inside the nucleus

(Polyakov, Physics Letters B555, 2003)

- Beam Charge Asymmetry observed on Hydrogen and Deuterium

- Beam Spin Asymmetry observed on heavy targets ranging from Deuterium up to Xenon

$\rightarrow$ Separate coherent and incoherent fractions

- Final period of HERMES dedicated to exclusive processes like DVCS

$\rightarrow$ Installation of Recoil Detector 


\section{The HERMES Recoil Detector}

- Surround unpolarised gas target

- Detect recoiling nucleon

$\rightarrow$ momentum range $>0.25 \mathrm{GeV} / \mathrm{c}$

- Measure complete final state

$\rightarrow$ suppress semi-inclusive background

$\rightarrow$ distinguish intermediate resonances $(\Delta)$

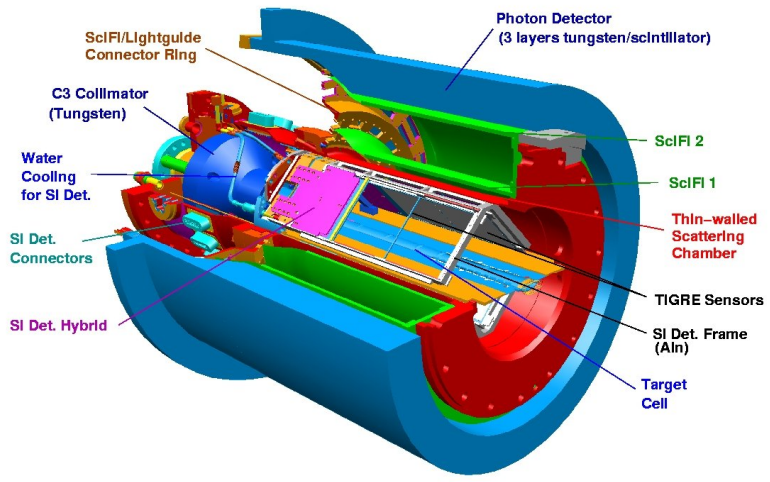

- Currently being commissioned 